ISSN 1112-9867

lable online at

http://www.jfas.info

\title{
SIMULATION OF THERMO-ELASTICS PROPERTIES OF THERMAL BARRIER COATINGS
}

\author{
A. M. Ferouani*, H. I. Faraoun and H. Aourag* \\ Laboratoire d'Étude et Prédiction des Matériaux, Unité de Recherche Matériaux et \\ Energies Renouvelables, Faculté des sciences, Département de physique, université de \\ Tlemcen, B-P119 Tlemcen 13000, Algérie
}

Received: 10 February 2011 / Accepted: 08 June 2010 / Published online: 30 June 2011

\begin{abstract}
Thermal barrier coatings are used to protect different parts in compressors and turbines from heat. They are generally composed of two layers, one metallic layer providing resistance to heat corrosion and oxidation, and one thermally insulating ceramic layer. Two different techniques are industrially used. Plasma spray results in a lamellar structure granting a low thermal conductivity, but with a low thermal expansion compliance. Electron Beam Physical Vapour Deposition generates a columnar structure allowing a better accommodation of the thermal expansion stresses, entailing improved lifetime of the coating, but with a higher thermal conductivity.

The aim of the paper presented here is to develop a procedure of analysis based on the micro structural observation for the prediction of the properties of new coatings in court of industrial development and to predict the effect of the posterior thermal treatment on the properties of the coatings carried out. For a given coating, one has to calculate linear elasticity and its evolution with the temperature as well as thermal expansion, aiming at predicting different parameters related to the in service deterioration.
\end{abstract}

Key words: thermal barriers, thermal projection, micrographics (MEB), Finite element method, code OOF

Author Correspondence, e-mail: madjid_phy@yahoo.fr

ICID: 1020771 


\section{INTRODUCTION}

Les barrières thermiques pour aubes représentent un saut technologique et constituent la seule façon à moyen terme d'accroître significativement performances et durées de vie des turbines aéronautiques. Elles sont des revêtements céramiques, déposés sur les parties métalliques des turbomachines aéronautiques ou terrestres. Leur but est d'isoler thermiquement les parties chaudes des turbines en maintenant la température de surface des structures métalliques inférieure à celle de leur environnement gazeux. La faible conductivité thermique de ces revêtements permet d'accroître la température de fonctionnement des moteurs et ainsi d'améliorer leur rendement. Actuellement, les systèmes les plus performants sont constitués de revêtements de zircone partiellement stabilisée à l'Yttrine - un matériau qui présente de bonnes propriétés mécaniques telle qu'une haute résistance à la propagation des fissures, et donc à la fatigue thermomécanique. Sa conductivité thermique est très faible, proche de celle des verres (de 1 à $2 \mathrm{~W} \cdot \mathrm{m}^{-1} \cdot \mathrm{K}^{-1}$ ). Son coefficient de dilatation thermique est relativement élevé $\left(10.10^{-6} \mathrm{~K}^{-1}\right)$, voisin de celui des alliages à base de nickel (de l'ordre de $\left.14.10^{-6} \mathrm{~K}^{-1}\right)$.

La simulation à l'échelle de la microstructure, par la méthode des éléments finis, offre la possibilité de simuler l'effet de l'arrangement microstructural sur les propriétés d'intérêt de ce type de revêtements. Le but du présent travail est de développer une procédure d'analyse basée sur l'observation microstructurale pour la prédiction des propriétés de nouveaux revêtements en cour de développement industriel et, éventuellement, qui permettrait de prédire l'effet du traitement thermique postérieur à l'élaboration sur les propriétés des revêtements réalisés.

Le code du domaine public d'analyse par éléments finis - OOF (de l'anglais Object Oriented Finite Element Analysis) permet, sur la base d'images microscopiques (réelles ou simulées), de calculer pour un revêtement donné l'élasticité linéaire et son évolution avec la température ainsi que l'expansion thermique. Notre idée est de faire usage des possibilités d'analyse offertes par ce code, 

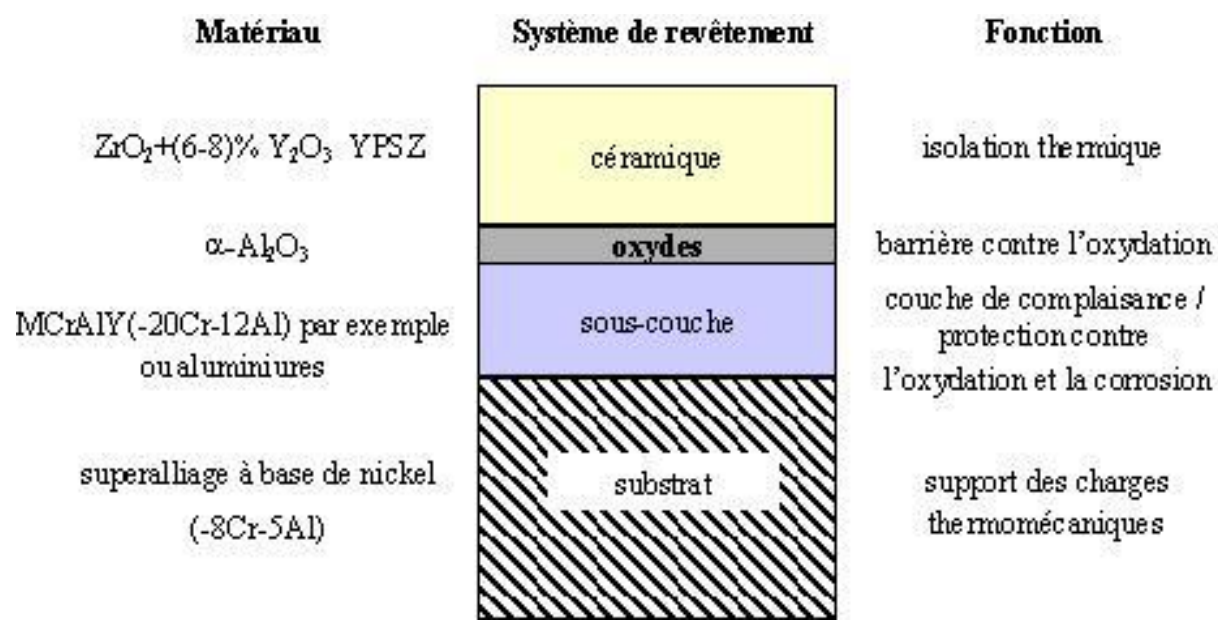

Fig.1. Système barrière thermique typique - matériaux sélectionnés et fonctions associées [1].

\section{LA TECHNIQUE DE CALCUL}

L'information microstructurale requise dans notre analyse est acquise sous la forme d'une image numérique bidimensionnelle. En fait, l'importance des images numériques dans la science est qu'elles permettent une analyse quantitative. La méthode des éléments finis est essentiellement un moyen de convertir un ensemble d'équations différentielles partielles en un ensemble d'équation algébriques approximatives. La théorie sous-jacente est assez simple :

L'énergie élastique emmagasinée dans un pixel est donnée par :

$$
\mathrm{E}_{\mathrm{n}}=\frac{1}{2} \int \mathrm{d}^{3} \mathrm{r} \varepsilon_{\mathrm{pq}} \mathrm{C}_{\mathrm{Pqrs}} \varepsilon_{\mathrm{rs}}
$$

Où les déformations $\varepsilon_{p q}$ et les modules d'élasticités $C_{p q r s}$ sont sous forme tensorielle, p, q, r et s étant égales à $\mathrm{x}$ ou $\mathrm{y}$, et l'intégrale porte sur tout le volume du pixel. L'énergie totale est obtenue par la sommation sur tous les pixels du système. Du moment que le tenseur de déformations est symétrique, une notation plus simple est souvent utilisée, la notation de Voigt. La déformation est un vecteur des trois composantes indépendantes $\left(\varepsilon_{x x}, \varepsilon_{y y}, \varepsilon_{x y}\right)$,

et $C_{p q r s}$ est écrit sous la forme plus compacte $C_{\alpha \beta}$ donnée par :

$$
\varepsilon_{p p}=\frac{\partial u_{p}}{\partial X_{p}} ; \varepsilon_{p q}=\frac{\partial u_{p}}{\partial X_{q}}+\frac{\partial u_{q}}{\partial X_{p}}
$$

de sorte que l'équation de l'énergie se transforme en : 


$$
\mathrm{E}_{\mathrm{n}}=\frac{1}{2} \int \mathrm{d}^{3} \mathrm{r} \varepsilon_{\alpha} \mathrm{C}{ }_{\alpha \beta} \varepsilon_{\beta}
$$

avec $\alpha$ et $\beta=1,2$ ou $3(1=x x, 2=$ yy et $3=x y)$.

Pour résoudre le système ainsi défini, on introduit ses variables globales :

$\mathrm{U}_{m p}$ : la composante $\mathrm{p}$ du vecteur déplacement du noeud $\mathrm{m}$,

$C_{\alpha \beta}$ : le tenseur des modules d'élasticité (en notation de Voigt) pour un pixel (égal à zéro dans le cas des pixels présents dans les zones représentant des porosités),

$E=\left(E_{x x}, E_{y y}, E_{x y}\right):$ La contrainte élastique totale appliquée au système,

$\varepsilon=\left(\varepsilon_{x x}, \varepsilon_{y y}, \varepsilon_{x y}\right):$ La contrainte locale en un point $(\mathrm{x}, \mathrm{y})$ dans le pixel,

$D_{r p s q}$ : la matrice de rigidité dans le pixel.

La discrétisation de l'équation (2) se traduit dans ces conditions par l'équation :

$$
\mathrm{E}_{\mathrm{n}}=\frac{1}{2} \mathrm{u}^{\mathrm{T}}{ }_{\mathrm{rp}} \mathrm{D}_{\mathrm{rpsq}} \mathrm{U}_{\mathrm{sq}}
$$

La minimisation par la méthode du gradient conjugué permet, par la suite, la résolution dusystème en retrouvant, pour une déformation externe donnée, le système Contraintes/déformations locales qui minimise l'énergie totale.

\section{DETAILS DE L'APPLICATION}

Les géométries bidimensionnelles générées par le traitement d'images sont appropriées pour une analyse par la méthode des éléments finis. La génération du maillage a été, pour ces calculs, effectuée en utilisant le code OOF (de l'anglais : Object Oriented Finite Element Analysis) [2]. La procédure de maillage employée dans ce code génère des éléments de taille décroissante sur l'interface des régions de couleurs différentes, permettant ainsi de bien séparer les contours des différentes phases. La figure 2. Présente les différentes étapes nécessaires pour la réalisation d'un maillage sur une micrographie type. 

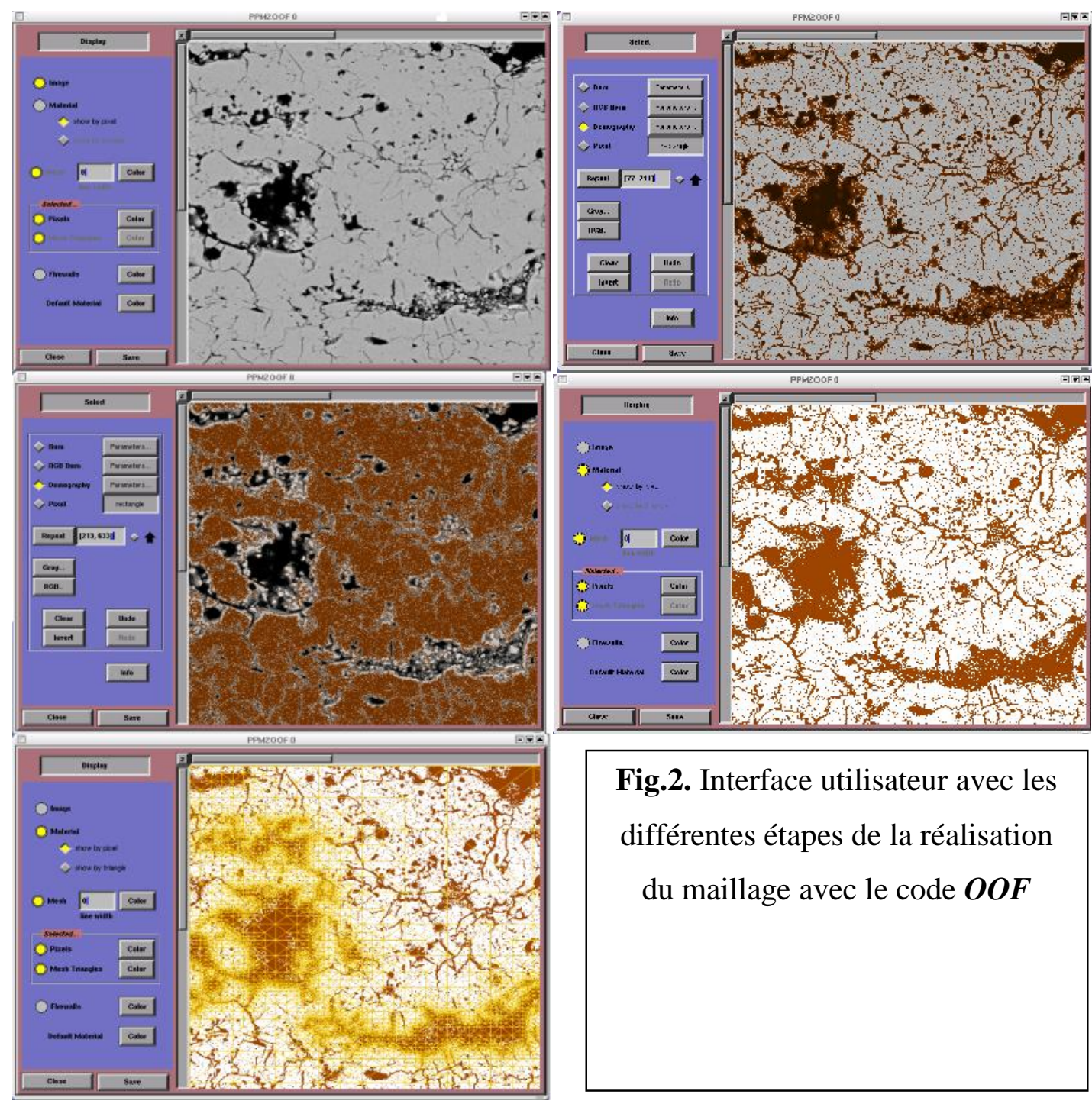

Fig.2. Interface utilisateur avec les différentes étapes de la réalisation du maillage avec le code $\boldsymbol{O O F}$

\section{LES RESULTATS ET DESCUTION}

LE CALCUl Du MOdule DE YOung : Un revêtement de Zircone Yttrirée $\left(\mathrm{ZrO}_{2}-\mathrm{Y}_{2} \mathrm{O}_{3}\right)$ a été élaboré par projection thermique puis traité thermiquement pour une durée de 100 heures à $1200^{\circ} \mathrm{C}$. Nous disposons de deux ensembles d'images numériques représentant les micrographies obtenues par microscopie électronique à balayage (MEB). Le premier ensemble correspond à l'échantillon brut (directement après la projection) et le second à l'échantillon après le traitement thermique. 


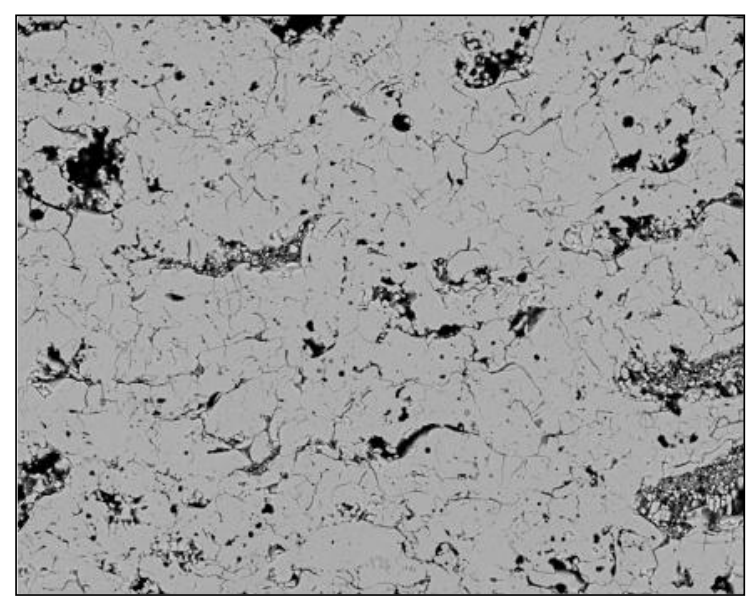

(a)

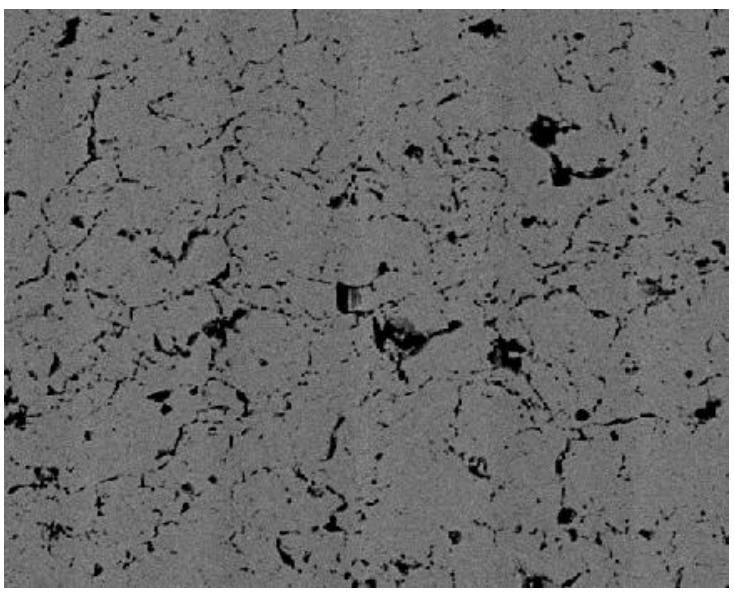

(b)

Fig.3. Micrographies du revêtement analysé. (a) avant et (b) après le traitement thermique.

Toutes les micrographies sont d'abord converties en des images binaires où la couleur blanche représente la matrice de zircone et la couleur noire représente la porosité (voire figure

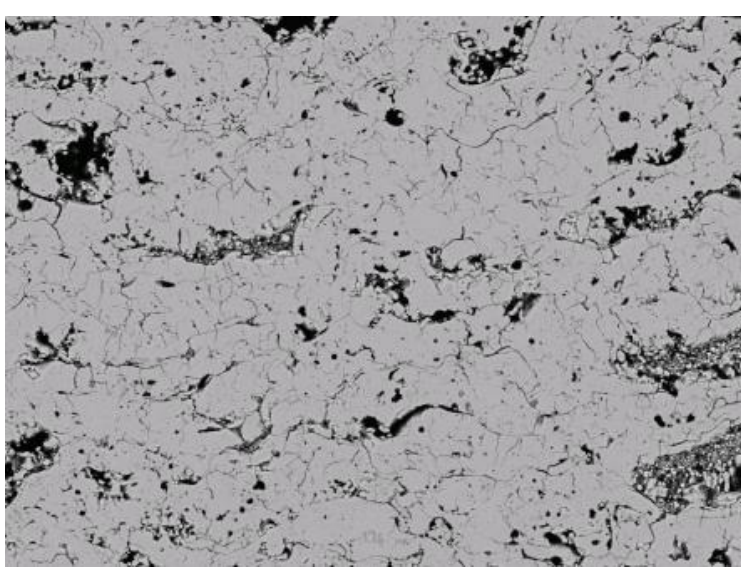

(a)
$4)$.

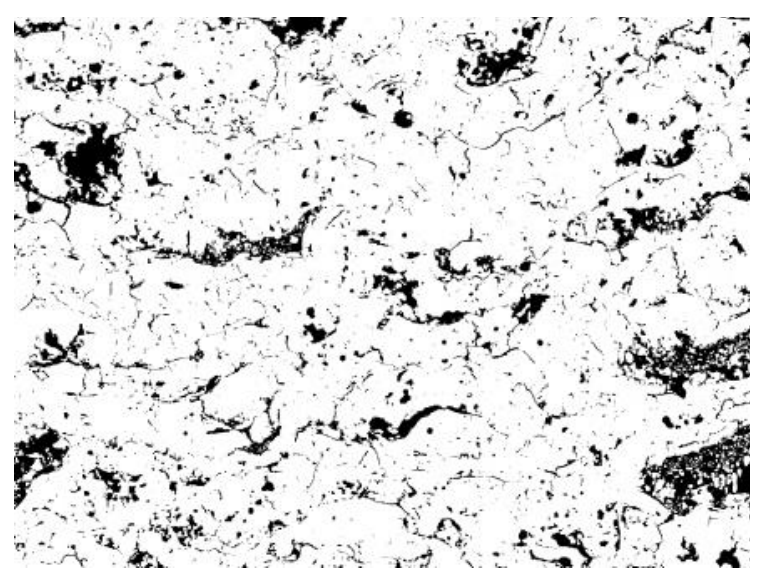

(b)

Fig.4. Binarisation des micrographies.

La grille est ensuite construite à l'aide du sous-programme de maillage et le calcul du module de Young est effectué dans l'approximation de la loi de Hook. Dans l'approximation bidimensionnelle, nous obtenons : 


$$
\left(\begin{array}{c}
\varepsilon_{x} \\
\varepsilon_{y} \\
\varepsilon_{x y}
\end{array}\right)=\left(\begin{array}{ccc}
\frac{1}{E_{x}} & -\frac{v_{x}}{E_{x}} & 0 \\
-\frac{v_{y}}{E_{y}} & \frac{1}{E_{y}} & 0 \\
0 & 0 & \frac{1}{G}
\end{array}\right) \times\left(\begin{array}{c}
\sigma_{x} \\
\sigma_{y} \\
\sigma_{x y}
\end{array}\right), \text { avec } \frac{v_{x}}{E_{x}}=\frac{v_{y}}{E_{y}}
$$

Où $\sigma$ et $\varepsilon$ représentent la contrainte et la déformation respectivement; E est le module de Young, $v$ le coefficient de Poisson et G le module de cisaillement. La direction y est celle qui est perpendiculaire au dépôt et est de ce fait celle qui nous intéresse. Nos calculs sont de ce fait restreins au calcul du module de Young dans la direction y en utilisant l'équation (5). Dans la pratique, le calcul est effectué en appliquant une déformation unidirectionnelle $\left(\varepsilon_{\mathrm{i}} \neq 0\right.$ et $\varepsilon_{\mathrm{j}}=0$ pour $\left.\mathrm{j} \neq \mathrm{i}\right)$ et en calculant la contrainte ainsi générée.

Pour chacun des deux ensembles d'images, notés A et B pour le revêtement avant et après traitement thermique respectivement, la moyenne est calculée sur les résultats des calculs effectués sur les séries d'images. Les Résultats obtenus du module de Young pour les deux séries de micrographies (A) et (B) et présentés sur le graphique de la figure 5 .

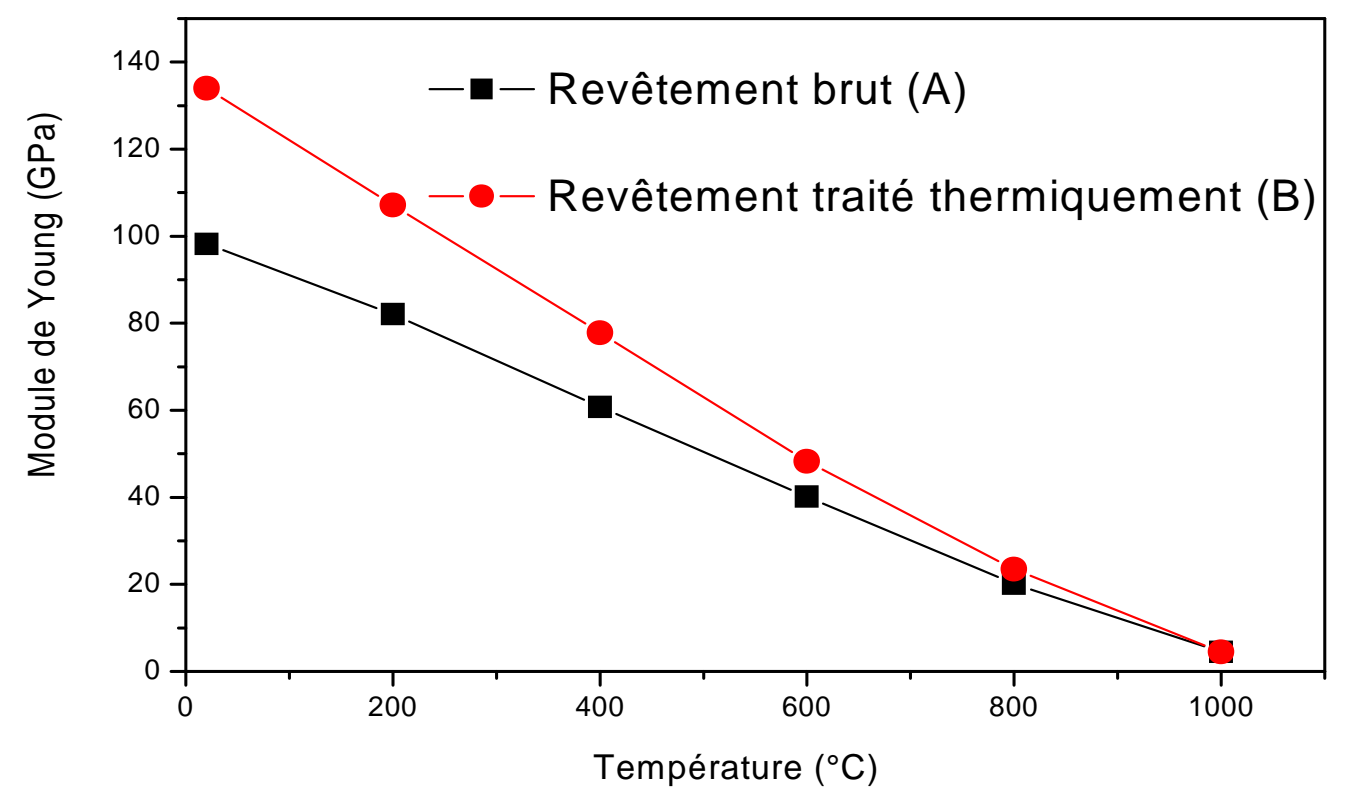

Fig.5. Variation du module de Young calculé en fonction de la température 
D'un autre côté, ce qui est immédiatement visible sur la courbe, est que le module de Young diminue avec la température. Cet effet est bien logique est cohérent avec le fait établi expérimentalement. En effet, tout matériau perd de sa cohésion intrinsèque lorsque la température augmente suite au relâchement des liaisons inter atomiques lorsque les vibrations thermiques augmentent. Cependant, il y a un autre aspect tout aussi important dans la diminution de la dureté, en l'occurrence la modification de la microstructure. Lorsqu'il s'agit de matériaux métalliques, l'augmentation de la température favorise la croissance des grains et le mouvement des dislocations, ce qui rend les matériaux métalliques plus malléables, et donc moins durs, à haute température. Dans notre cas, il s'agit d'une céramique avec une microstructure assez particulière dans laquelle l'aspect dominant est la porosité. Ainsi, l'analyse de l'effet de température sur les propriétés élastique de ce revêtement doit passer par l'analyse de l'effet de la température sur la porosité.

\section{L'EFFET DE LA POROSITE}

Le revêtement étudié est un dépôt réalisé à l'aide de la technique de projection thermique.

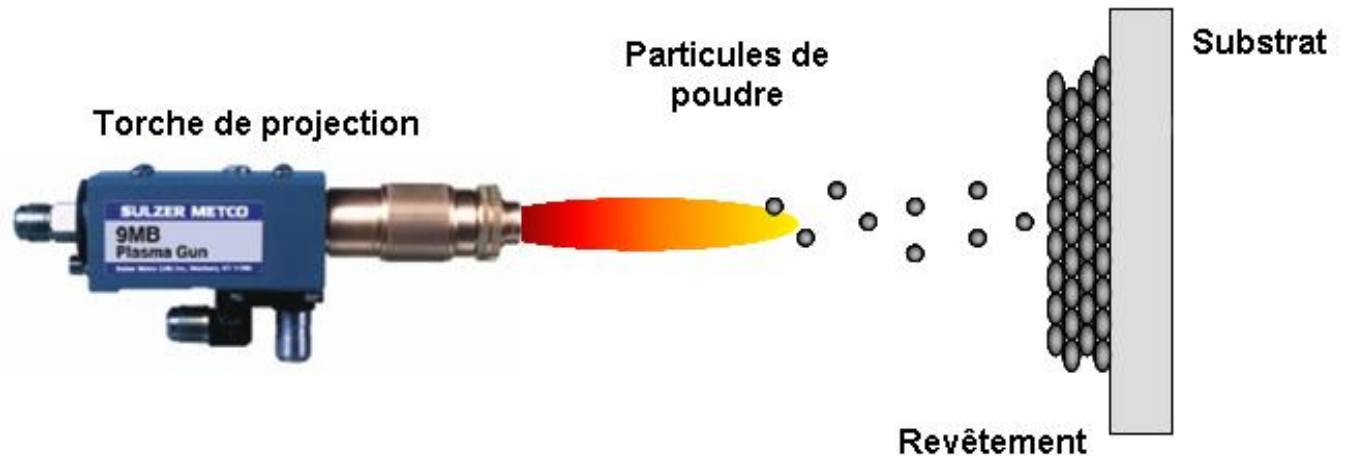

Fig.6. Schéma de principe de la projection thermique [3].

La porosité d'un milieu est la fraction du volume vide $\mathrm{V}_{\text {vide }}$ sur le volume total $\mathrm{du}$ milieu, aussi appelé volume apparent $\mathrm{V}_{\text {app}}$, elle dépend en générale de la forme et de la taille des particules.

$$
\mathrm{p}=\frac{\mathrm{V}_{\text {vide }}}{\mathrm{V}_{\text {app }}}
$$

La porosité entraîne toujours une baisse du module de Young par rapport au matériau céramique. Plusieurs modèles basés sur des développements théoriques ou issus de l'expérience permettent de décrire cette décroissance [4,5]. Citons, à titre d'exemples, 
les approches classiques de Hasselman [6] et Mackenzie [7] à partir de modèles de sphères isolées dans une matrice, la loi empirique proposée par Spriggs [8],

Ce modèle permet de relier le module de Young d'un matériau à la valeur de son taux de porosité suivant l'équation suivante :

$$
\mathrm{E}=\mathrm{E}_{0} \exp (-\mathrm{b} . \mathrm{P})
$$

Où $\mathrm{E}$ et $\mathrm{E}_{0}$ sont les modules de Young du matériau poreux et du matériau massif respectivement. La constante $\mathrm{b}$ dépend du type du matériau analysé et est déterminée empiriquement. $\mathrm{P}$ est le taux de porosité mesuré.

La figure7 présente l'histogramme de variation de la porosité calculée en fonction de la température. Nous pouvons voir qu'à basse température le taux de porosité est visiblement réduit grâce au traitement thermique. De ce fait résulte l'augmentation du module de Young de l'échantillon. Ceci peut être expliqué par la compaction de la matière : en éliminant les pores, le matériau devient plus dense et donc plus dur.

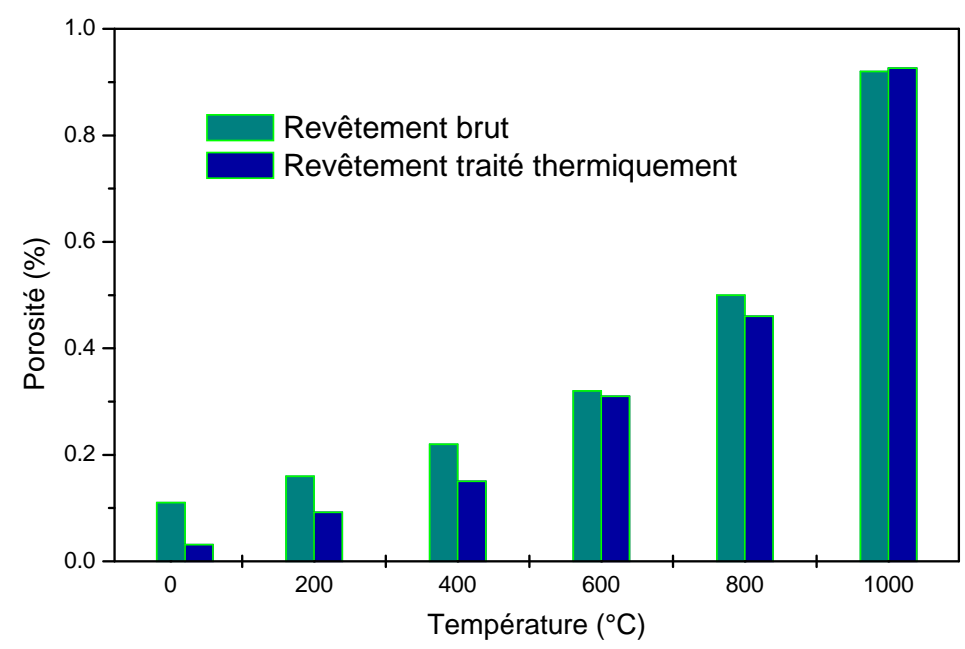

Fig.7. Évolution de la porosité en fonction de la température pour le revêtement brut et le revêtement après traitement thermique

En augmentant la température, le taux de porosité augmente dans les deux cas. La figure 8. montre l'effet de l'augmentation de la température sur la microstructure du revêtement. L'effet est quelque peu exagéré par l'application des conditions aux limites libres. 


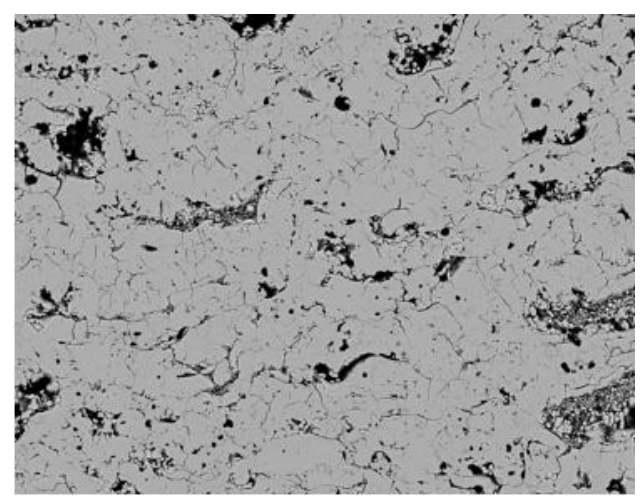

(a)

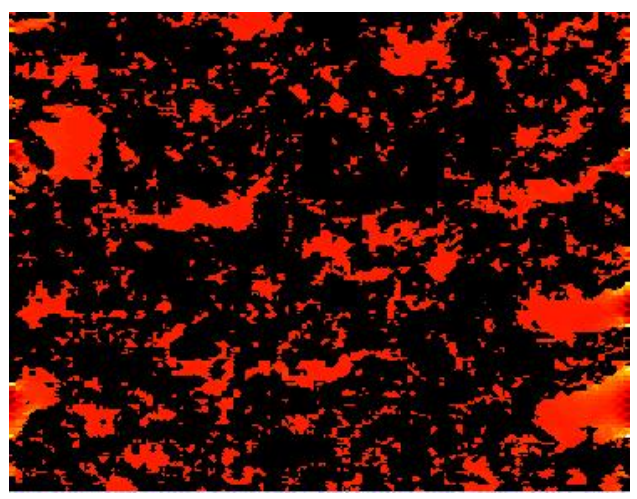

(b)

Fig.8. Effet de la température sur la microstructure du revêtement. (a) Micrographie du revêtement à la température ambiante. (b) Microstructure résultante du modèle à la température virtuelle de $1000^{\circ} \mathrm{C}$.

\section{L'EFFET DES FISSURES :}

La figure 9 montre bien que les fissures présentes dans le revêtement sont bien visibles sur les micrographies électroniques.

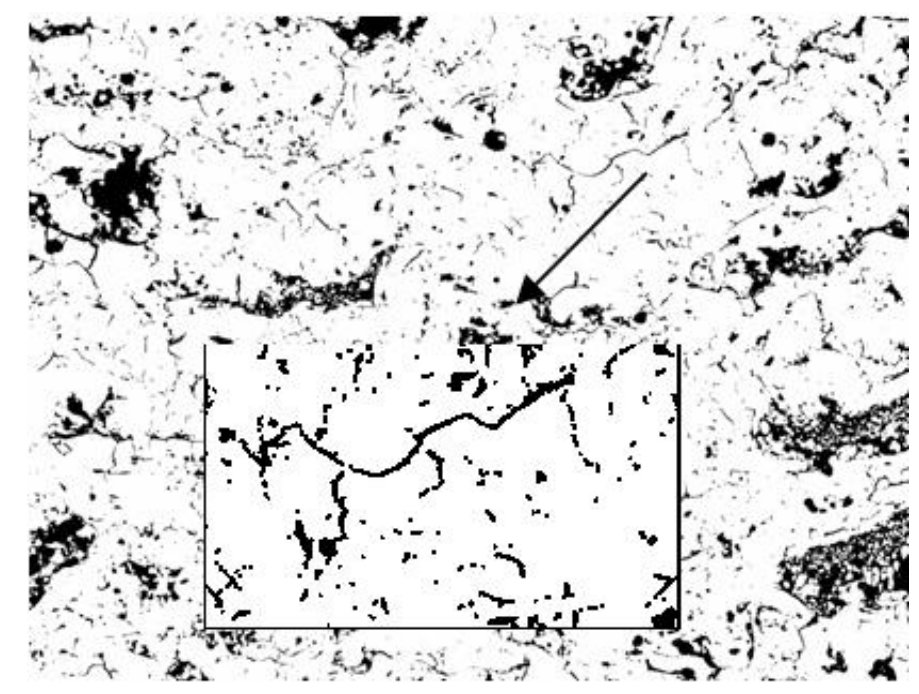

Fig.9. Les fissures dans le revêtements

D’une façon générale, la variation relative du module de Young peut être utilisée pour évaluer l'endommagement d'un matériau, en utilisant par exemple la relation de Kachanov [9,10] : 


$$
\mathrm{D}=1-\frac{\mathrm{E}}{\mathrm{E}_{0}}
$$

Où $\mathrm{D}$ est le paramètre d'endommagement, $\mathrm{E}_{0}$ et $\mathrm{E}$ les modules de Young du matériau massif et du matériau endommagé respectivement.

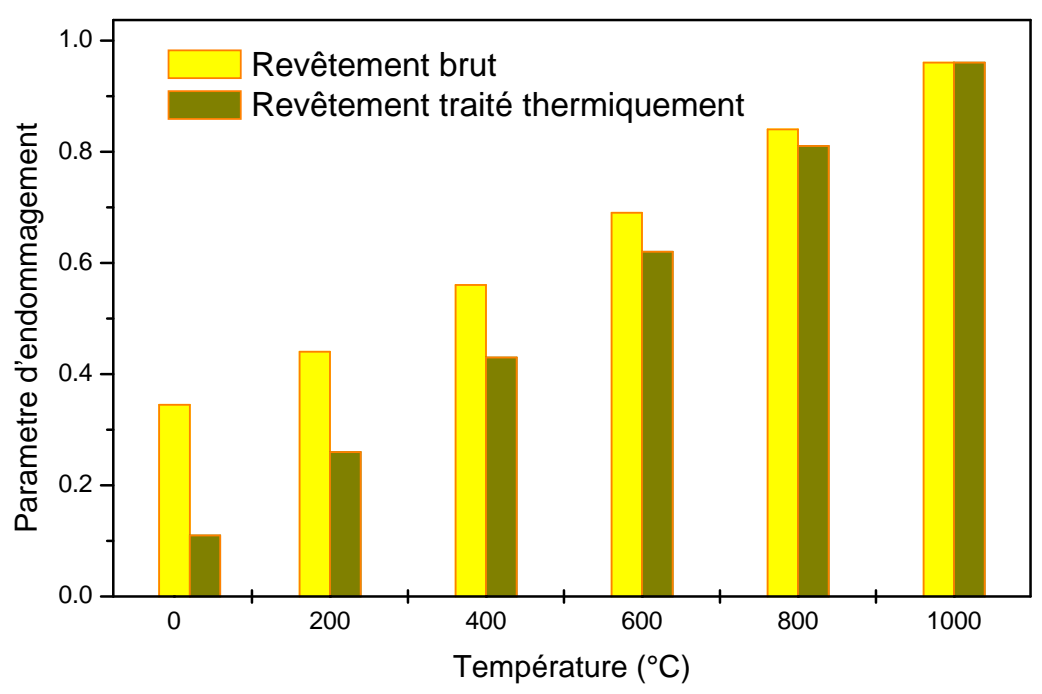

Fig.10. Evolution du paramètre d'endommagement en fonction de la température

Nous pouvons voir que nos calculs permettent de prédire que le paramètre d'endommagement du revêtement a nettement baissé après qu'on l'ait traité thermiquement. Lors du service par contre, l'élévation de température réduira cette amélioration et le comportement du revêtement sans traitement thermique sera quasi identique à celui du revêtement traité.

5. LE COEFFICIENT DE DILATATION THERMiQue : Le coefficient de dilatation thermique $\alpha$ représente la pente de la courbe $\Delta \mathrm{L} / \mathrm{L}=\mathrm{f}(\Delta \mathrm{T})$. Où $\mathrm{L}$ et $\Delta \mathrm{L}$ sont respectivement la longueur de l'échantillon et la variation due à l'augmentation de la température. Cette variation est d'ordinaire linéaire et $\alpha$ peut être calculé en évaluant la pente de la droite.

Techniquement, pour calculer $\alpha$, nous utilisant les mêmes images binaires, avec un maillage un peu plus fin. Aucune contrainte mécanique extérieure n'est imposée et les conditions aux limites libres sont appliquées pour permettre à l'échantillon de se dilater librement. La température globale de l'échantillon est en suite variée et les déformations linéaires relatives sont obtenues. Le coefficient de dilatation thermique linéaire est sera : 


$$
\alpha=\frac{1}{\mathrm{~L}} \frac{\Delta \mathrm{L}}{\Delta \mathrm{T}}
$$

La figure 11 présente un exemple des courbes de variation de la longueur en fonction de la température.

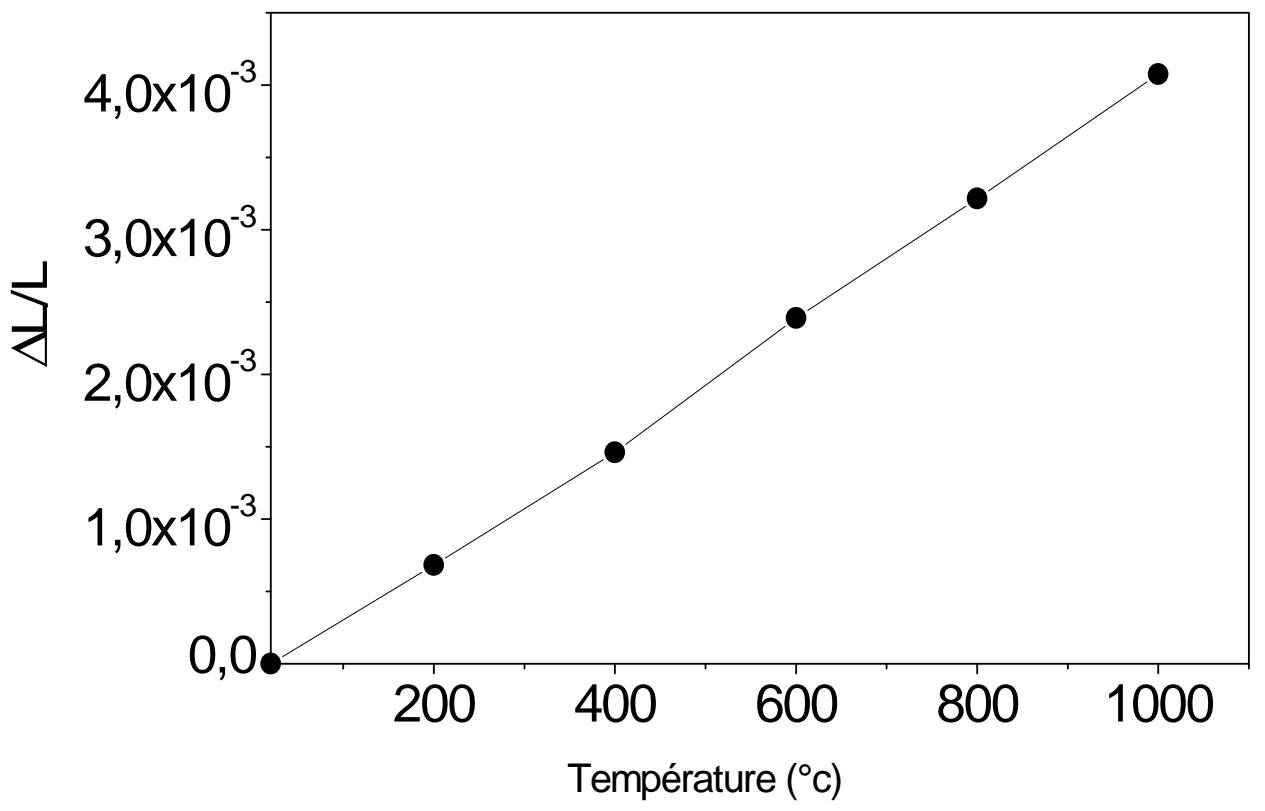

Fig.11. Variation en fonction de la température de la longueur de l'échantillon

A l'aide de cette méthode, le coefficient de dilatation thermique a été évalué pour différentes valeurs de la température pour les deux séries de micrographies. Les résultas sont présentés sur la figure 12 . 


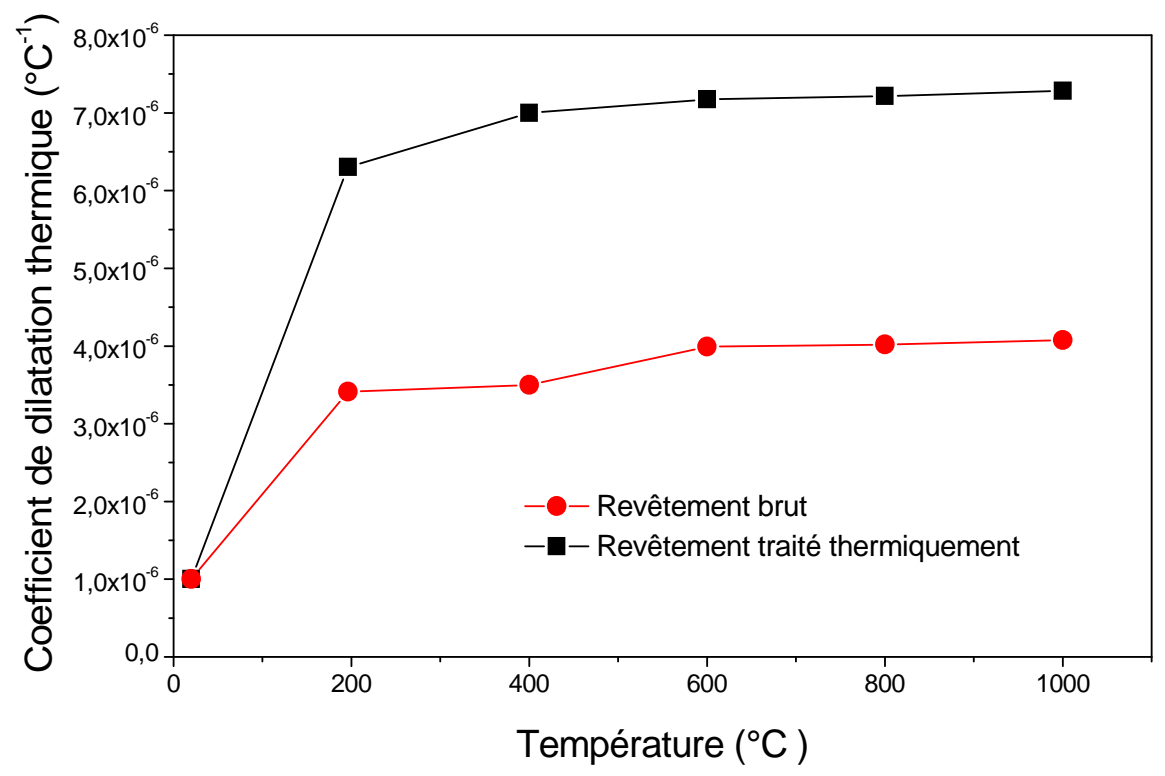

Fig.12. Valeurs du coefficient de dilatation thermique calculé pour le revêtement brut et le revêtement traité thermiquement

Nous pouvons voir que le coefficient de dilatation thermique augmente très rapidement avec l'augmentation de la température entre 20 et $200^{\circ} \mathrm{C}$. Ensuite, sa valeur se stabilise rapidement dans les deux cas.

Nous pouvons aussi constater que la valeur du coefficient de dilatation thermique est supérieure dans le cas du revêtement qui a subi le traitement thermique. La dilatation thermique à l'échelle macroscopique est un phénomène qui se propage de proche en proche, et est ainsi largement dépendant de la présence de porosités et de fissures dans la microstructure. Comme le traitement thermique diminue le taux de porosité, et densifie le revêtement, il est donc plausible que le coefficient de dilatation thermique soit amélioré par ce même procédé.

\section{Conclusion}

Dans ce travail, notre objectif était de montrer qu'à partir de micrographies obtenues par microscopie électronique (MEB) il est possible d'examiner le comportement thermoélastique d'un revêtement. Il s'agit en l'occurrence d'un revêtement de zircone yttriée réalisé par projection thermique (plus particulièrement la projection plasma) qui 
a subit un traitement thermique postérieur. Nous avons comparé les propriétés calculées sur une série de micrographies de ce revêtement avant et après le traitement thermique.

Nos calculs sont réalisés à l'aide de la technique des éléments finis, où le maillage de base est réalisé sur les micrographies qui contiennent l'information sur la microstructure du revêtement. Nous avons déterminé le module de Young et le coefficient de dilatation thermique pour les deux ensembles d'images numériques qui représentent les micrographies obtenues par microscopie électronique sur ce revêtement avant et après le traitement thermique.

\section{REFERENCES}

[1] U.Schulz, C.Leyens, K.Fritscher, M.Peters, B.Saruhan-Brings, O. Lavigne, J-M. Dorvaux, M. Poulain, R. Mévrel, M. Caliez Some recent trends in research and technology of advanced thermal barrier coatings Aerospace Science and Technology Volume 7, Issue 1, 2003, p73-80.

[2] National Institute of Standards and Technology (NIST), OOF: Analysis of Real Material Microstructures (http://www.ctcms.nist.gov/oof/).

[3] HERMAN, « Les revêtements par projection plasma », Pour la science, 133, p. 4855,1988

[4] J. B. Wachtman Jr., "Elastic deformation of ceramics and other refractory materials," in Mechanical and Thermal Properties of Ceramics National Bureau of Standards Special Publication $\mathrm{N}^{\circ}$ 303, J. B. Wachtman Jr., (Washington: National Bureau of Standards, 1969), pp. 139-168.

[5] J. B. Wachtman Jr., "Elastic deformation of ceramics and other refractory materials," in Mechanical and Thermal Properties of Ceramics National Bureau of Standards Special Publication $\mathrm{N}^{\circ}$ 303, J. B. Wachtman Jr., (Washington: National Bureau of Standards, 1969), pp. 139-168.

[6] R. W. Rice, "Microstructural dependence of mechanical behaviour of ceramics," in Treatise on materials science and technology, vol. 11: Properties and microstructure R. K. MacCrone, (New York: Academic Press, 1977), pp. 199-381

[7] D. P. H. Hasselman, "On the porosity dependence of elastic moduli of polycrystalline refractory materials," J. Am. Ceram. Soc. Vol. 45, N 9 (1962): pp. 452 453. 
[8] R. M. Spriggs, "Expression for effect of porosity on elastic modulus of polycrystalline refractory materials, particularly aluminium oxide," J. Am. ceram. Soc. Vol. 44, No 12 (1961): pp. 628-629.

[9] L. M. Kachanov, "Time of the rupture process under creep conditions," Izv. Akad. Nauk. SSR. Otd. Tech. Nauk. Vol. 8 (1958): pp. 26-31.

[10] J. Lemaitre et J.-L. Chaboche, Mécanique des matériaux solides 2ème éd., (Paris: Dunod, 1996), pp. 343-444. 


\section{SIMULATION DES PROPRIETES THERMOELASTIQUES DES REVETEMENTS DE BARRIERES THERMIQUES}

\section{RESUME}

Les barrières thermiques sont utilisées pour protéger les pièces sous-jacentes dans un compresseur ou une turbine de la chaleur. Elles sont généralement constituées d'une sous-couche métallique résistante à l'oxydation et à la corrosion à chaud, et d'une couche céramique thermiquement isolante, usuellement de la zircone partiellement stabilisée à l'yttrine. Deux techniques sont utilisées au niveau industriel. La projection par plasma permet l'obtention de structures lamellaires présentant une faible conductivité thermique, mais accommodant peu les contraintes induites par la différence de dilatation entre le substrat et la zircone. Les dépôts en phase vapeur assistés par faisceau d'électrons résultent en une structure colonnaire permettant de mieux accommoder ces contraintes et ainsi d'étendre la durée de vie des dépôts, mais présentant une conductivité thermique plus élevée.

Les travaux présentés dans cet article ont pour but de développer une procédure d'analyse basée sur l'observation microstructurale pour la prédiction des propriétés de nouveaux revêtements en cours de développement industriel et de prédire l'effet du traitement thermique postérieur à l'élaboration sur les propriétés des revêtements réalisés. Pour un revêtement donné, il s'agit de calculer l'élasticité linéaire et son évolution avec la température ainsi que l'expansion thermique, pour déduire différents paramètres relatifs à l'endommagement durant le service.

Mots-clés : barrières thermiques, projection thermique, micrographies (MEB), La méthode des éléments finis, le code OOF

\section{How to cite this article}

Ferouani A M, Faraoun H I and Aourag H. Simulation of thermo-elastics properties of thermal barrier coatings. J Fundam Appl Sci. 2011, 3(1), 19-34. 